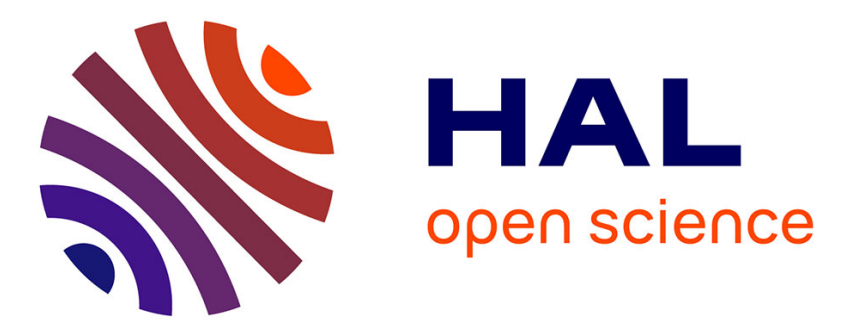

\title{
Novel Vector Sensors Design with Three Co-located or Distributed Elements for the 3D DoA Estimation
}

\author{
Jimmy Lominé, Christophe Morlaas, Hervé Aubert
}

\section{To cite this version:}

Jimmy Lominé, Christophe Morlaas, Hervé Aubert. Novel Vector Sensors Design with Three Colocated or Distributed Elements for the 3D DoA Estimation. Progress In Electromagnetics Research B, 2014, 57, pp.207 - 220. 10.2528/PIERB13102902 . hal-01108968

\section{HAL Id: hal-01108968 \\ https://hal-enac.archives-ouvertes.fr/hal-01108968}

Submitted on 23 Jan 2015

HAL is a multi-disciplinary open access archive for the deposit and dissemination of scientific research documents, whether they are published or not. The documents may come from teaching and research institutions in France or abroad, or from public or private research centers.
L'archive ouverte pluridisciplinaire HAL, est destinée au dépôt et à la diffusion de documents scientifiques de niveau recherche, publiés ou non, émanant des établissements d'enseignement et de recherche français ou étrangers, des laboratoires publics ou privés. 


\title{
Novel Vector Sensors Design with Three Co-located or Distributed Elements for the 3D DoA Estimation
}

\author{
Jimmy Lominé ${ }^{1,2, *}$, Christophe Morlaas ${ }^{3}$, and Herve Aubert ${ }^{4}$
}

\begin{abstract}
In this paper, two novel vector sensors using a reduced number of radiating elements are proposed to estimate the directions of arrival of incoming electromagnetic signals in the 3D space, azimuth and elevation angles. The first one uses co-located radiating elements while the other one is based on distributed antenna elements. These two sensors combine only two half-loops and one linear monopole placed on a metallic plate in view of embedded applications. Full wave electromagnetic simulations are performed to take into account the electromagnetic coupling effects between the antenna elements. The directions of arrival estimation accuracy of electromagnetic signals incoming in arbitrary directions in the full 3D space are computed from the MUSIC algorithm. For experimental validation purpose, a prototype is manufactured and the directions of arrival measurements are performed. Then a novel vector sensor design with a reduced number of antenna elements is presented. The antenna elements are spatially distributed. An analysis is carried out to determine the largest distance between the antenna elements without causing ambiguous estimations in the 3D space. The estimation accuracy of the resulting sensor is reported. Finally the performances of these two vector sensors are compared.
\end{abstract}

\section{INTRODUCTION}

The direction of arrival (DoA) estimation is present in different applications such as radar, radio astronomy, sonar and navigation $[1,2]$. The purpose is to estimate the DoA of incoming electromagnetic (EM) signals transmitting by sources located at unknown positions. It can be used for defense applications by detecting and localizing enemy transmissions or for civil applications by finding emergency beacons for Search And Rescue (SAR). In order to determine the DoA of incoming EM signals, two strategies are usually applied: the use of the spatial diversity $[2]$ and the use of the polarization diversity [3]. A combination of these two well-known techniques could also be performed [49]. Nowadays many direction finding algorithms use the spatial diversity such as interferometry [10], which consists of measuring the phase differences between radiating elements of the sensor when it is illuminated by an incoming EM signals. In principle this approach can be applied to the 3D DoA estimation but the design of the sensors is not easy when it is applied to DoA in the full 3D space. The application of the polarization diversity requires smaller sensors because it does not need a minimum distance between radiating elements to estimate the DoA of incoming signals [11]. The DoA is estimated through the measurement of the EM field components of the incoming signal. The spatial distribution, combined with the diversity of polarization, has the advantage to improve the accuracy of the DoA estimation [8] and also, to decrease the undesirable mutual coupling between the antenna elements [9]. Following [3] the polarization diversity can be applied by using a vector sensor of six co-located antenna elements, such as three orthogonal electric dipoles and three orthogonal magnetic dipoles [11], in order to measure the six components of the incoming EM field.

\footnotetext{
Received 29 October 2013

* Corresponding author: Jimmy Lominé (jlomine@rockwellcollins.com).

${ }^{1}$ Rockwell Collins France, 6 av. Didier Daurat, B.P. 20008, Blagnac 31701, France. ${ }^{2}$ INP Toulouse, 6 allée Emile Monso, B.P. 34038 , 31029 Toulouse cedex 4, France. ${ }^{3}$ ENAC/TELECOM EMA, 7 av. E. Belin, Toulouse 31055 , France. ${ }^{4}$ University of Toulouse; UPS, INSA, INP, ISAE; LAAS; 7 av. du colonel Roche, Toulouse F-31077, France.
} 
For vector sensors embedded on vehicles, the metallic objects that surrender the antennas can alter the measurement of the field components. However, the measurement of less than six components can be sufficient to estimate the DoA of an incoming transverse magnetic (TM) signal from regions that are not hidden by the surrounding metallic objects. Thus only three co-located antenna elements combining two orthogonal magnetic dipoles and one electric dipole may be sufficient for the DoA estimation of TM incoming signals from the upper half-space [12-18]. However, to the authors' knowledge, no vector sensor using only three distributed antenna elements for polarization diversity applied to the 3D DoA estimation (azimuth and elevation angles) has been reported yet. Very recently Slater et al. [19] have investigated a vector sensor design with linearly distributed elements but only the 2D DoA estimation (azimuth angles only) has been reported.

In this paper two compact sensors combining only three dipoles are proposed for the 3D DoA estimation (azimuth and elevation angles). The first one (sensor A) uses co-located radiating elements while the second one (sensor B) combines distributed antenna elements. These two sensors incorporate two orthogonal loops and one dipole placed on a metallic plate in view of embedded applications. The DoA accuracy is computed and measured using the MUltiple SIgnal Classification (MUSIC) algorithm reported in [20].

This article is organized as follows. The Section 2 introduces the electromagnetic analysis of vector sensor using a reduced number of antenna elements. The design of a vector sensor with co-located radiating elements is reported in the Section 3 and the DoA accuracy is computed. This accuracy is measured in an anechoic chamber to validate the simulation approach. An original compact vector sensor using only three distributed antennas is proposed in the Section 4. A criterion based on the orthogonality between the signal and noise subspaces is proposed to determine the largest separation distance between constitutive elements of the sensor without causing ambiguous estimations. Finally the DoA accuracy obtained with the proposed sensors are compared.

\section{DATA MODEL OF THE VECTOR SENSOR COMBINING TWO MAGNETIC DIPOLES AND ONE ELECTRIC DIPOLE}

The analysis of a vector sensor combining six antenna elements is presented. From this analysis the data model of the sensor with a reduced number of radiating elements, combining only two magnetic dipoles and one electric dipole, is derived and the DoA estimation of incoming EM waves is introduced. In this section, mutual coupling between the antenna elements is neglected.

\subsection{Data Model of a Vector Sensor Measuring the Six Components of the Incoming EM Fields}

When the polarization diversity approach is applied to determine the DoA of an incoming signal, a vector sensor is generally used to simultaneously measure the six components of the EM field. Theoretically these components can be measured by using three orthogonal magnetic dipoles and three orthogonal electric dipoles. The data model of such vector sensor is given as follows [11]:

$$
\underbrace{\left[\begin{array}{l}
E_{x}(\phi, \theta, \gamma, \eta) \\
E_{y}(\phi, \theta, \gamma, \eta) \\
E_{z}(\phi, \theta, \gamma, \eta) \\
H_{x}(\phi, \theta, \gamma, \eta) \\
H_{y}(\phi, \theta, \gamma, \eta) \\
H_{z}(\phi, \theta, \gamma, \eta)
\end{array}\right]}_{A(\phi, \theta, \gamma, \eta)}=\underbrace{\left[\begin{array}{cc}
\cos \phi \cos \theta & -\sin \phi \\
\sin \phi \cos \theta & \cos \phi \\
-\sin \theta & 0 \\
-\sin \phi & -\cos \phi \cos \theta \\
\cos \phi & -\sin \phi \cos \theta \\
0 & \sin \theta
\end{array}\right]}_{g(\phi, \theta)} \times \underbrace{\left[\begin{array}{c}
\sin \gamma e^{j \eta} \\
\cos \gamma
\end{array}\right]}_{p(\gamma, \eta)} \odot \underbrace{\left[\begin{array}{c}
e^{-j \vec{k}(\phi, \theta) \cdot \vec{r}_{E_{x}}} \\
e^{-j \vec{k}(\phi, \theta) \cdot \vec{r}_{E_{y}}} \\
e^{-j \vec{k}(\phi, \theta) \cdot \vec{r}_{E_{z}}} \\
e^{-j \vec{k}(\phi, \theta) \cdot \vec{r}_{H_{x}}} \\
e^{-j \vec{k}(\phi, \theta) \cdot \vec{r}_{H_{y}}} \\
e^{-j \vec{k}(\phi, \theta) \cdot \vec{r}_{H_{z}}}
\end{array}\right]}_{d(\phi, \theta)}
$$

where $E_{x}, E_{y}$ and $E_{z}$ denote to the three components of the electric field and, $H_{x}, H_{y}$ and $H_{z}$ denote to the three components of the magnetic field in the Cartesian $(X Y Z)$ coordinate system. $A(\phi, \theta, \gamma, \eta)$ is called the steering vector. Each component depends on the azimuth angle $\phi \in[0 ; 2 \pi]$, the elevation angle $\theta \in[0 ; \pi / 2]$ (refer to Figure 1 for the definition of the azimuth and elevation angles) and the 


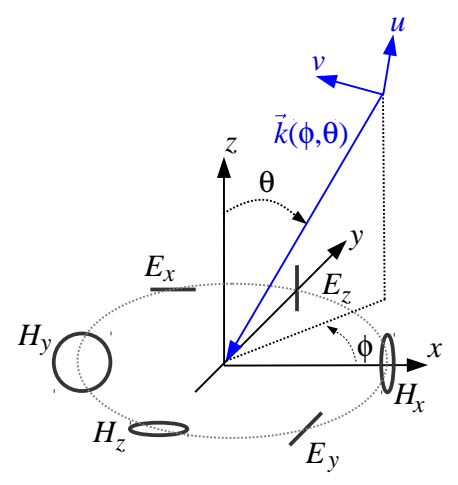

Figure 1. Vector sensor representation with three magnetic dipoles and three electric dipoles $(\phi$ : azimuth angle; $\theta$ : elevation angle).

polarization vector $p(\gamma, \eta)$. In the $(u, v)$ polarization plane, orthogonal to the free space wave vector $\vec{k}(\phi, \theta)$ (see Figure 1), the electric field is defined as follows:

$$
\vec{E}(t)=E_{0} \sin (\gamma) \cos (w t-\vec{k} \cdot \vec{r}) \cdot \hat{u}+E_{0} \cos (\gamma) \cos (w t-\vec{k} \cdot \vec{r}+\eta) \cdot \hat{v}
$$

where $E_{0}$ is the norm of the field vector $\vec{E}(t), \gamma \in[0 ; \pi / 2]$ denotes the auxiliary polarization angle, $\eta \in[-\pi ; \pi]$ denotes the polarization phase difference and $w$ the radial frequency. Thus the polarization vector $p$ is defined in terms of two angles: $\gamma$ and $\eta$. The angles $\phi$ and $\theta$ define the DoA of the incoming EM signal and consequently denote the angles to be derived from the measurement of all field components by the vector sensor. This derivation may be performed using specific direction finding algorithms such as MUSIC [20] or ESPRIT [21]. The $\odot$ operator in Eq. (1) denotes the elementwise multiplication operator and $d(\phi, \theta)$ designates the spatial phase shift vector due to an eventual spatial distribution of the radiating elements. This vector depends on the free space wave vector $\vec{k}(\phi, \theta)$ of the incoming EM wave and the vector position $\vec{r}_{i}$ of the $i$ th antenna elements. If the vector sensor is placed on the surface of a metallic support, some electromagnetic field components cannot be measured.

\subsection{Data Model of a Vector Sensor Measuring the $E_{z}, H_{x}$ and $H_{y}$ Components of the Incoming EM Fields}

When a sensor is located on the surface of a metallic plate in the $x O y$ plane, the components $E_{x}, E_{y}$ and $H_{z}$ cannot be measured on this surface. Thus the vector sensor consists only of a combination of two magnetic dipoles, one to measure the component $H_{x}$ and the other to measure the component $H_{y}$, and one electric dipole to measure the component $E_{z}$. The data model Eq. (1) can then be reduced to:

$$
\underbrace{\left[\begin{array}{l}
E_{z}(\phi, \theta, \gamma, \eta) \\
H_{x}(\phi, \theta, \gamma, \eta) \\
H_{y}(\phi, \theta, \gamma, \eta)
\end{array}\right]}_{A_{r}(\phi, \theta, \gamma, \eta)}=\underbrace{\left[\begin{array}{cc}
-\sin \theta & 0 \\
-\sin \phi & -\cos \phi \cos \theta \\
\cos \phi & -\sin \phi \cos \theta
\end{array}\right]}_{g_{r}(\phi, \theta)} \times p(\gamma, \eta) \odot \underbrace{\left[\begin{array}{l}
e^{-j \vec{k}(\phi, \theta) \cdot \vec{r}_{E_{z}}} \\
e^{-j \vec{k}(\phi, \theta) \cdot \vec{r}_{H_{x}}} \\
e^{-j \vec{k}(\phi, \theta) \cdot \vec{r}_{H_{y}}}
\end{array}\right]}_{d_{r}(\phi, \theta)}
$$

If the incoming EM waves are transverse electric (TE) $(\eta=0$ and $\gamma=0)$ or transverse magnetic (TM) $(\eta=0$ and $\gamma=\pi / 2)$, the data model can be reduced to Eq. (4) and Eq. (5) respectively as shown in [18]:

$$
\begin{aligned}
& A_{\mathrm{TE}}(\phi, \theta)=\left[\begin{array}{c}
0 \\
-\cos \phi \cos \theta \\
-\sin \phi \cos \theta
\end{array}\right] \odot d_{r}(\phi, \theta) \\
& A_{\mathrm{TM}}(\phi, \theta)=\left[\begin{array}{c}
-\sin \theta \\
-\sin \phi \\
\cos \phi
\end{array}\right] \odot d_{r}(\phi, \theta)
\end{aligned}
$$


where $A_{\mathrm{TE}}$ and $A_{\mathrm{TM}}$ denote the steering vector of the reduced vector sensor in the case of the horizontal and the vertical polarization, respectively. In this paper, only incoming EM signals with vertical polarization are considered, and consequently $A(\phi, \theta, \gamma, \eta)=A_{\mathrm{TM}}(\phi, \theta)$. The dipole will be used to estimate the elevation angles of the DoA and the two loops will be used to estimate the azimuth angles. This data model does not allow the computation of the Poynting vector of the incoming EM field because the components $E_{x}, E_{y}$ and $H_{z}$ are not measured. Consequently a DoA direction finding algorithm based on the Poynting vector is not applicable in this case.

In this paper, the MUSIC algorithm is used to estimate the DoA of a vertically-polarized incoming EM signals from the data model:

$$
X(\phi, \theta, \gamma, \eta, t)=A(\phi, \theta, \gamma, \eta) S(t)+n(t)
$$

where $X(\phi, \theta, \gamma, \eta, t)$ is the measured snapshots at the sensor ports, $S(t)$ the incoming EM signal and $n(t)$ the uncorrelated noise assumed to be gaussian with a zero-mean and a variance $\sigma^{2}$. This algorithm is based on the concept of signal and noise subspaces [20]. The noise subspace is derived from the eigenvalue decomposition from the covariance matrix of the snapshots $X(\phi, \theta, \gamma, \eta, t)$. Then the signal subspaces are projected on the noise subspace, and the DoA angle is determined by the lowest projection value which is caused by the orthogonality between both subspaces. The quality of the noise subspace computation and consequently the accuracy of the DoA estimation, depend on the number of snapshots and the noise level [22]. The signal-to-noise ratio (SNR) at each antenna input port varies according to the amplitude variation of each field component in the full 3D space. Therefore, a reference signal-to-noise ratio (RSNR) for an arbitrary direction $\left(\phi_{r e f}=0^{\circ}, \theta_{r e f}=0^{\circ}\right.$, in this paper) and for only one antenna input port is defined to compute the receiver noise power $P_{N}$ as follows:

$$
P_{N}=\left|X^{(i)}\left(\phi_{\text {ref }}, \theta_{\text {ref }}, t\right)\right|^{2} / \mathrm{RSNR}
$$

where $X^{(i)}(\phi, \theta, t)$ denotes the $i$ th element of the vector $X(\phi, \theta, t)$ and RSNR the reference signal-tonoise ratio. For a given $P_{N}$ the SNR at each antenna port can be deduced as follows:

$$
\operatorname{SNR}^{(i)}(\phi, \theta, t)=\left|X^{(i)}(\phi, \theta, t)\right|^{2} / P_{N}
$$

where $\operatorname{SNR}^{(i)}(\phi, \theta, t)$ denotes the signal-to-noise ratio at the $i$ th antenna input port for the DoA $(\phi, \theta)$. In this paper, the selected port is the loop port used to measure the $H_{y}$ component.

\section{SENSOR A: THREE CO-LOCATED ANTENNA ELEMENTS FOR MEASURING $E_{z}, H_{x}$ AND $H_{y}$}

The vector sensor A combines two loops and one linear dipole to measure the components $E_{z}, H_{x}$ and $H_{y}$. For this sensor, the antenna elements are co-located and consequently $d_{r}(\phi, \theta)$ is a unit vector.

\subsection{DoA Estimation Results through Analytic Approach}

A first simulation of DoA estimation is performed by neglecting the EM coupling between radiating elements. The closed-form equations of elementary dipoles radiation are used in this simulation to compute the signal received at each antenna input port. The incoming signals are assumed to be incident plane waves. The RSNR is set to $15 \mathrm{~dB}$. A number $(N)$ of 100 snapshots has been used in the MUSIC algorithm. Moreover the average of the estimation accuracy has been obtained by using a number $(I T)$ of 100 estimations per DoA. Various angles of arrival of incoming signals have been selected between $45^{\circ}$ and $135^{\circ}$ in azimuth to assess the DoA estimation errors. Outside this angular range, the accuracy can be predicted due to the symmetry of the sensor geometry. The simulation results are shown in Table 1.

The accuracy of the DoA estimation is excellent for azimuth angles due to the absence of EM coupling between the antenna elements. However the estimation error of the elevation angle reaches $5^{\circ}$ when $\theta$ is close to $90^{\circ}$. This error is intrinsic to the sensor A because $\theta$ is estimated mainly from 
Table 1. Mean estimation error of the sensor A - Simulation results without EM coupling between the antenna elements $-\mathrm{RSNR}=15 \mathrm{~dB}, N=100$ and $I T=100$.

\begin{tabular}{|c|c|c|c|}
\hline$\phi$ & $\theta$ & Mean azimuth error & Mean elevation error \\
\hline $55^{\circ}$ & $20^{\circ}$ & $0^{\circ}$ & $0^{\circ}$ \\
\hline $95^{\circ}$ & $40^{\circ}$ & $0^{\circ}$ & $0.35^{\circ}$ \\
\hline $130^{\circ}$ & $60^{\circ}$ & $0^{\circ}$ & $1.2^{\circ}$ \\
\hline $45^{\circ}$ & $80^{\circ}$ & $0^{\circ}$ & $5^{\circ}$ \\
\hline
\end{tabular}

the knowledge of the $E_{z}$ component (see the steering vector $A_{\mathrm{TM}}$ ). In Figure 2, the variation of $A_{\mathrm{TM}}$ according to $\theta$ is analyzed through the power $P_{r}(\theta)$ received by the electric dipole and computed from (9).

$$
P_{r}(\theta)=20 \log \left(\frac{\left|A_{\mathrm{TM}}^{(1)}(\theta)\right|}{\left|\max \left(A_{\mathrm{TM}}^{(1)}(\theta)\right)\right|}\right)
$$

where ${ }^{(1)}$ denotes the first component of the steering vector $A_{\mathrm{TM}}$. In the angular range from $\theta=60^{\circ}$ to $\theta=90^{\circ}$, the maximum dynamic of the received power is $1.25 \mathrm{~dB}$ while it is $11.3 \mathrm{~dB}$ for the elevation angles included between $\theta=10^{\circ}$ and $\theta=40^{\circ}$. The higher the variation in an angular range, the higher the accuracy of the estimation. It correlates with the results given in Table 1. Beside, as shown in Figure 2, the SNR decreases for $\theta$ lower than $10^{\circ}$ and consequently the DoA estimation deteriorates.

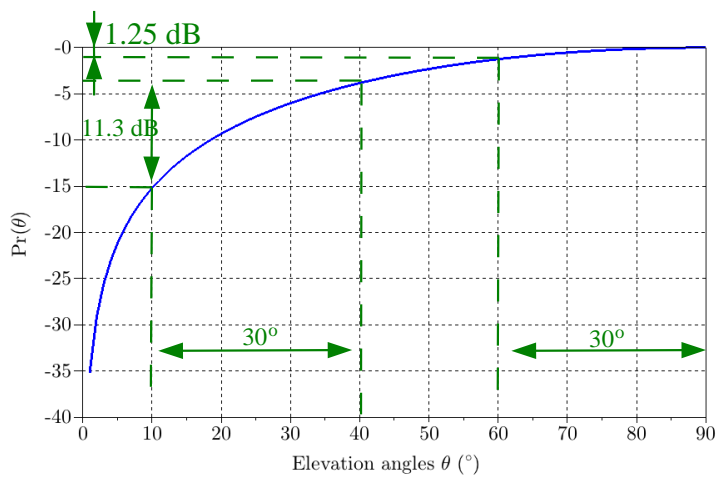

Figure 2. Normalized $P_{r}(\theta)$ at the input port of the electric dipole in the $E$-plane - simulation results.

In pratice eventual sensor anomalies or mutual coupling between dipoles may affect the DoA estimation accuracy. To take into account these effects, full wave electromagnetic simulations have been performed and the results are reported in Section 3.3.

\subsection{Antenna Design}

The vector sensor A, shown in Figure 3 has been modeled and simulated with the electromagnetic simulation software HFSS. The operating frequency has been set to $2.8 \mathrm{GHz}$. The sensor dimensions are given in Figure 5 and in Table 2. The antenna elements are printed on double layers FR4 PCB with a thickness of $1.5 \mathrm{~mm}$, a relative permittivity $\epsilon_{r}=4.4$ and a loss tangent of 0.02 . The double layers PCB used for the horizontal support is the ROGER4003 with a thickness of $1.542 \mathrm{~mm}, \epsilon_{r}=3.55$ and loss tangent of 0.0027. A metallic disc is placed at the bottom of the sensor $\mathrm{A}$ in view of embedded applications. Its diameter is fixed to $300 \mathrm{~mm}(\approx 2.8 \lambda)$. This metallic plate allows to reduce the size of the antenna elements by using the image theory (the loops are replaced by half-loops and the dipole by a monopole). Capacitive effects are performed to increase the electric length of the radiating elements and to get the impedance matching as shown in Figure 4. 


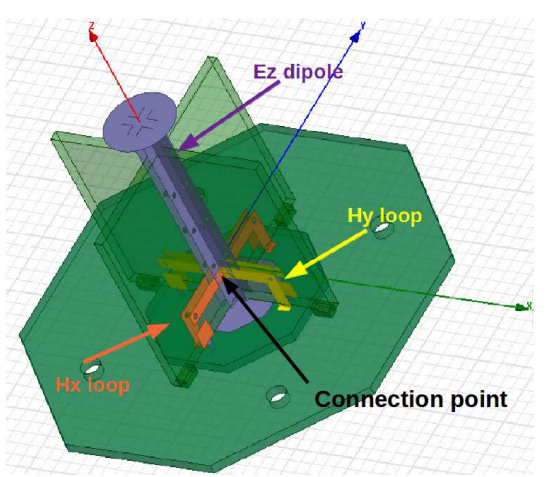

Figure 3. Design of the vector sensor A.

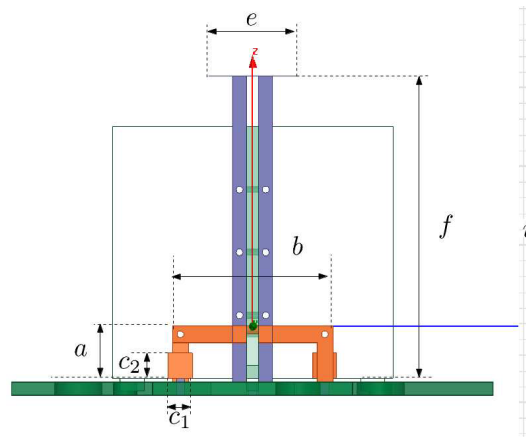

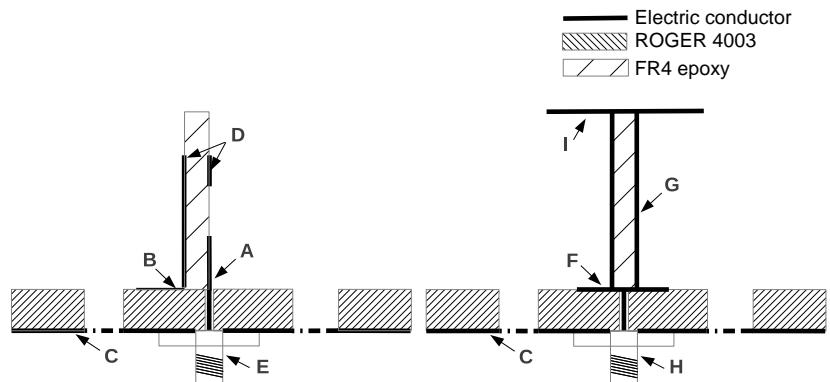

(a)

Figure 4. (a) Outline of the loop feeding. (b) Outline of the monopole feeding (A, B, C, $\mathrm{D}, \mathrm{E}, \mathrm{F}, \mathrm{G}, \mathrm{H}$ and $\mathrm{I}$ are defined in the following paragraph).

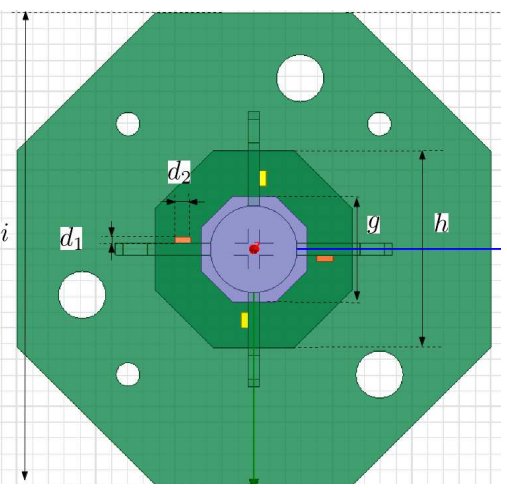

Figure 5. Vector sensor A combining three co-located radiating elements: two orthogonal half-loops and one linear monopole.

Table 2. Dimensions of the sensor A (in mm).

Antenna design

\begin{tabular}{|c|c|c|c|c|c|c|c|c|c|c|c|}
\hline$a$ & 6.74 & $c_{1}$ & 3 & $d_{1}$ & 0.8 & $e$ & 11 & $g$ & 13.41 & $i$ & 60 \\
\hline$b$ & 20 & $c_{2}$ & 3 & $d_{2}$ & 2 & $f$ & 36.5 & $h$ & 25 & & \\
\hline
\end{tabular}

The loop feeding is performed through a coupling effect between the metallic plate A connected to the SMA connector E and the loop D. This coupling effect allows to set the antenna input impedance. A second capacitive effect, between the plates $\mathrm{B}$ and $\mathrm{C}$, is used to adjust the resonant frequency at the required value. Since the half-loops are printed on a double PCB layers, the same process is applied to the other half-loops extremity. A phase shift of $\pi$ is applied to the opposite feeding to generate a constant current distribution all along the half-loops. For the monopole G, there are two capacitive effects. The first one is in parallel to the feeding port connected to the SMA connector H. It uses to adjust the impedance matching. It is performed through the coupling between the metallic plates $\mathrm{F}$ and $\mathrm{C}$. The second one is located at the end of the monopole with the metallic disc I. It uses to increase the electric length of the radiating element. All antenna elements are connected together at the point indicated in Figure 3.

\subsection{DoA Estimation Results through Full Wave Electromagnetic Simulations}

In order to estimate the DoA of incoming EM signals in the presence of the EM coupling between antenna elements, incident plane waves have been considered by using full wave electromagnetic simulations. The 
resulting steering vectors have been extracted and imported into the MUSIC algorithm. A gaussian noise is added to these data to create the snapshots $X(\phi, \theta, \gamma, \eta, t)$. The eventual EM coupling between the sensor antennas can alter the estimation accuracy. These effects have to be compensated with a calibration process [23]. Several calibration methods have been proposed in litterature [23-25]. In this work, it is directly performed through full wave electromagnetic simulations. The sensor is illuminated by an incident plane wave for each direction in the $3 \mathrm{D}$ half-space. An angular step of $5^{\circ}$ in azimuth and elevation has been chosen for limiting the use of high memory ressources. For each DoA, the steering vector is extracted and stored in a matrix, called "calibration matrix". The eventual amplitude and phase distortions are taken into account by substituting the antenna model used in the MUSIC algorithm by the calibration matrix. Thus the calibration process is ideal for the DoA estimation through full wave electromagnetic simulations.

The estimation configuration in the MUSIC algorithm are: RSNR $=15 \mathrm{~dB}, N=100$ and $I T=100$. The considered DoA of the incoming waves are those used in the analytic approach. The simulation results, given in Table 3, indicate that the sensor A allows estimating the DoA with an error of $4.8^{\circ}$. This DoA estimation error is due to the lack of dynamics of the power received at the monopole port as explained in the Section 3.1. The error is low and close to the theoretical prediction indicated in Table 1. The estimation of the DoA $(\phi, \theta)=\left(55^{\circ}, 20^{\circ}\right)$ is performed with an error of $1.7^{\circ}$. This is explained by a low SNR value at the monopole input port due to the low levels of the received signals for this elevation angle. This field attenuation is increased in the presence of the metallic plate: this plate alters the received field in this DoA as shown in the simulated radiation patterns in Figure 7. Thus the estimation of the elevation angles is less robust to the noise in this area. In these graphs, the elevation angles are on the radial axis and the azimuth angles on the circular axis.

Table 3. Mean estimation error by using the sensor A - simulation results by taking into account EM coupling between the antenna elements $-\mathrm{RSNR}=15 \mathrm{~dB}, N=100$ and $I T=100$.

\begin{tabular}{|c|c|c|c|}
\hline$\phi$ & $\theta$ & Mean azimuth error & Mean elevation error \\
\hline $55^{\circ}$ & $20^{\circ}$ & $0.8^{\circ}$ & $1.7^{\circ}$ \\
\hline $95^{\circ}$ & $40^{\circ}$ & $0.05^{\circ}$ & $0.5^{\circ}$ \\
\hline $130^{\circ}$ & $60^{\circ}$ & $0.2^{\circ}$ & $0.4^{\circ}$ \\
\hline $45^{\circ}$ & $80^{\circ}$ & $2.1^{\circ}$ & $4.8^{\circ}$ \\
\hline
\end{tabular}

\subsection{DoA Estimation Results through Experimentations}

The purpose of these experimentations is to validate the design methodology and the simulation results relative to the sensor A. A prototype of the sensor A, with an operating frequency set to $2.8 \mathrm{GHz}$, has been manufactured for this experimental validation as shown in Figure 6. The measurements are performed in an anechoic chamber. The calibration matrix obtained from the full wave electromagnetic simulation derived in the Section 3.3 is used into the MUSIC algorithm for this experimentation. In this case, the calibration process is no more ideal because the calibration matrix is determined from simulations and not from measurement. The DoA estimation is firstly performed by mean of full wave electromagnetic simulation. The corresponding results are summerized in the Table 4 . Then the measurement of the DoA estimation is reported in Table 5. Both estimations are performed with the same configuration: $\mathrm{RSNR}=30 \mathrm{~dB}, N=1$ and $I T=100$.

The simulation and measurement results are in good agreement. The highest errors are obtained for the same directions of arrival. The maximum measured errors of the DoA estimation are $9.2^{\circ}$ in azimuth and $7.5^{\circ}$ in elevation for $(\phi, \theta)=\left(55^{\circ}, 20^{\circ}\right)$. The estimation of the DoA $(\phi, \theta)=\left(55^{\circ}, 20^{\circ}\right)$ and $(\phi, \theta)=\left(45^{\circ}, 80^{\circ}\right)$ are not accurate, as expected from the simulation results. These errors are due to the low SNR in these directions. The measured radiation patterns are in good agreement with the simulated ones as shown in Figure 7. In these graphs, the elevation angles are on the radial axis and the azimuth angles on the circular axis. To improve the estimation accuracy in these DoA, the number of snapshots has to be increased (see Table 3). For the DoA $(\phi, \theta)=\left(95^{\circ}, 40^{\circ}\right)$ and $(\phi, \theta)=\left(130^{\circ}, 60^{\circ}\right)$, the estimation accuracy is close to the simulation results with an error lower than $5^{\circ}$ by measurement 


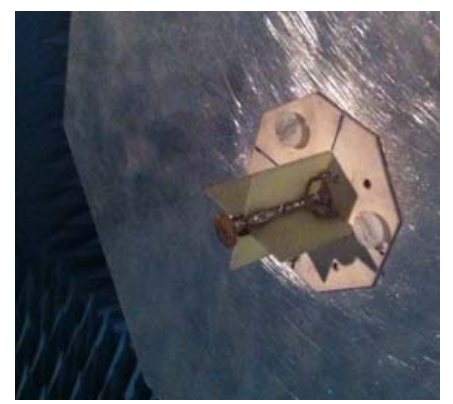

Figure 6. Prototype of the sensor A on a metallic plate of $300 \mathrm{~mm}$ in diameter.

Table 4. Simulated estimation error by using the sensor A with EM coupling - RSNR $=30 \mathrm{~dB}$ $N=1$ and $I T=100$.

\begin{tabular}{|c|c|c|c|}
\hline$\phi$ & $\theta$ & $\begin{array}{c}\text { Mean azimuth } \\
\text { error }\end{array}$ & $\begin{array}{c}\text { Mean elevation } \\
\text { error }\end{array}$ \\
\hline $55^{\circ}$ & $20^{\circ}$ & $7.4^{\circ}$ & $2.95^{\circ}$ \\
\hline $95^{\circ}$ & $40^{\circ}$ & $1.2^{\circ}$ & $2.75^{\circ}$ \\
\hline $130^{\circ}$ & $60^{\circ}$ & $1.35^{\circ}$ & $2.1^{\circ}$ \\
\hline $45^{\circ}$ & $80^{\circ}$ & $4.65^{\circ}$ & $5.9^{\circ}$ \\
\hline
\end{tabular}

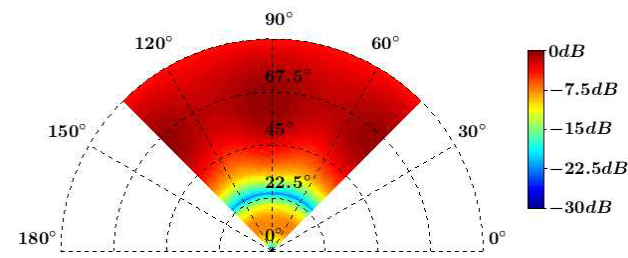

(a)

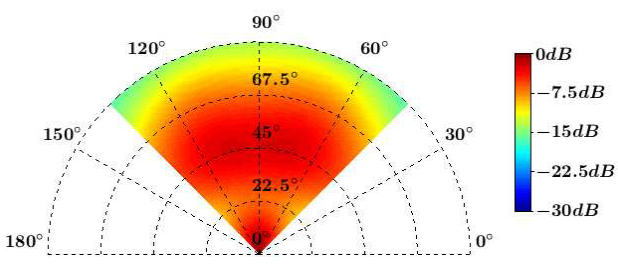

(c)

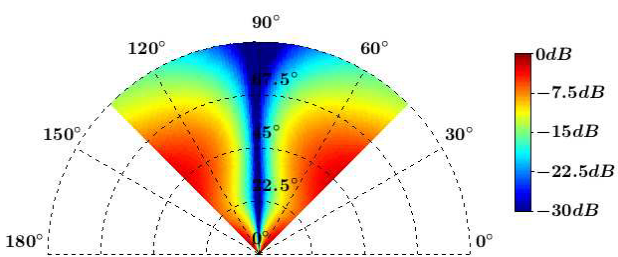

(e)
Table 5. Measured estimation error by using the sensor $\mathrm{A}-\mathrm{RSNR}=30 \mathrm{~dB}, N=1$ and $I T=100$.

\begin{tabular}{|c|c|c|c|}
\hline$\phi$ & $\theta$ & $\begin{array}{c}\text { Mean azimuth } \\
\text { error }\end{array}$ & $\begin{array}{c}\text { Mean elevation } \\
\text { error }\end{array}$ \\
\hline $55^{\circ}$ & $20^{\circ}$ & $9.2^{\circ}$ & $7.5^{\circ}$ \\
\hline $95^{\circ}$ & $40^{\circ}$ & $2.3^{\circ}$ & $5^{\circ}$ \\
\hline $130^{\circ}$ & $60^{\circ}$ & $3.6^{\circ}$ & $1.6^{\circ}$ \\
\hline $45^{\circ}$ & $80^{\circ}$ & $7.6^{\circ}$ & $2.8^{\circ}$ \\
\hline
\end{tabular}

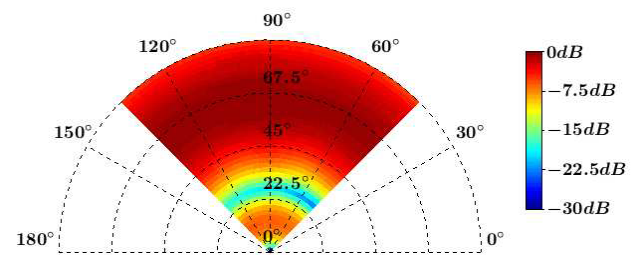

(b)

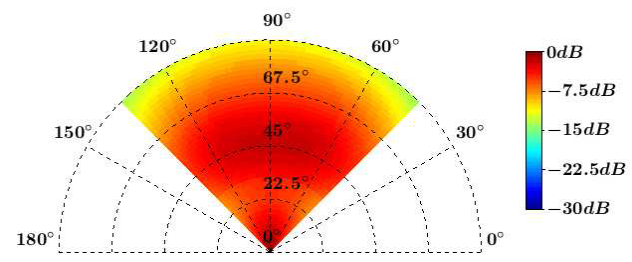

(d)

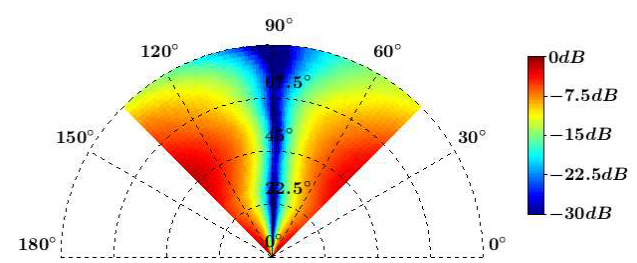

(f)

Figure 7. Normalized gain from $45^{\circ}$ to $135^{\circ}$ in azimuth and $0^{\circ}$ to $90^{\circ}$ in elevation of (a) the simulated monopole $E_{z}$, (b) the measured monopole $E_{z}$, (c) the simulated half-loop $H_{x}$, (d) the measured half-loop $H_{x}$, (e) the simulated half-loop $H_{y}$, (f) the measured half-loop $H_{y}$.

and $2.75^{\circ}$ by simulation. This small difference of accuracy is due to amplitude and phase distortions not taken into account in the simulated calibration matrix. 


\section{SENSOR B: THREE DISTRIBUTED ANTENNA ELEMENTS FOR MEASURING $E_{z}, H_{x}$ AND $H_{y}$}

In this section a vector sensor, called sensor B, with distributed antenna elements (i.e., not co-located) is proposed as shown in Figure 8. Compared with the co-located configuration of the sensor A, the spatial distribution of the radiating elements provides an additionnal phase shift at the antenna ports. This phase shift depends on the DoA of the incoming EM waves but also on the position of each antenna elements of the vector sensor. According to the electrical separation distance between the antenna elements and to the array geometry, some DoA estimations can be ambiguous. Thus to design the sensor B, a process allowing to spot ambiguous angles had to be implemented.

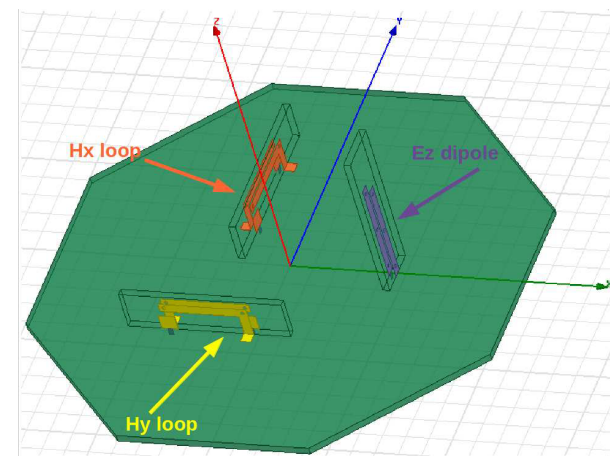

Figure 8. Design of the vector sensor B.

\subsection{Ambiguity Analysis Process}

The presence of ambiguous angles means that at least two steering vectors associated with two different DoA are orthogonal to the noise vector subspace [26]. The MUSIC algorithm computes the orthogonality between the signal vector subspaces and the noise vector subspace to estimate the DoA [20]. Thus this algorithm cannot distinguished two DoA with coplanar steering vectors. Before designing an antenna array applied to the DoA estimation, an analysis has to be carried out in order to define the largest separation distance between the antenna elements without causing ambiguous estimations. The angle between two steering vectors can be used as criterion to spot these ambiguities $[27,28]$. This angle is given by:

$$
\alpha\left(\phi_{1}, \theta_{1}, \phi_{2}, \theta_{2}\right)=\cos ^{-1} \frac{\left|A_{r}\left(\phi_{1}, \theta_{1}\right)^{*} \cdot A_{r}\left(\phi_{2}, \theta_{2}\right)\right|}{\left\|A_{r}\left(\phi_{1}, \theta_{1}\right)\right\| \cdot\left\|A_{r}\left(\phi_{2}, \theta_{2}\right)\right\|}
$$

where $\alpha \in[0 ; \pi / 2]$ denotes the angle (modulo $\pi / 2$ ) between the two steering vectors $A_{r}\left(\phi_{1}, \theta_{1}\right)$ and $A_{r}\left(\phi_{2}, \theta_{2}\right)$. Two coplanar steering vectors give $\alpha=0^{\circ}$ and two orthogonal steering vectors give $\alpha=90^{\circ}$.

\subsection{Antenna Design}

To determine the distance between the antenna elements of the sensor B, an ambiguity analysis is carried out. The range of the separation distance $r$ covered by this analysis starts from 0 (co-located elements) up to $\lambda / 2$. The radiating elements are equidistant of each others and located on a circle of $r / \sqrt{3}$ in radius. They are considered as elementary dipoles (magnetic and electric) and EM coupling effects are neglected. Since the sensor B is applied to the direction estimation in 3D half-space, the criterion $\alpha$, given in (10), is computed for $\phi_{1}$ and $\phi_{2} \in[0 ; 2 \pi]$ and for $\theta_{1}$ and $\theta_{2} \in[0 ; \pi / 2]$. For each DoA $\left(\phi_{1}, \theta_{1}\right)$, the minimum $\alpha$ is extracted to obtain the 3D ambiguity spectra given in Figure 9 . Up to a distance of $\lambda / 4$, no ambiguous angle is detected $\left(\alpha_{\min }\left(\phi_{1}, \theta_{1}\right)>0\right)$. However for $r$ close to $\lambda / 3$ an ambiguity appears between $\phi_{1}=0^{\circ}$ and $\phi_{1}=180^{\circ}$ for $\theta_{1}$ close to $90^{\circ}$ as shown in Figure $9(\mathrm{~d})$. This ambiguity shifts to the low $\theta_{1}$ when the separation distance increases as shown in Figures $9(\mathrm{e})$ and $9(\mathrm{f})$. A second ambiguity appears at $\lambda / 2$ between $\phi_{1}=90^{\circ}$ and $\phi_{1}=270^{\circ}$ for the $\theta_{1}$ close to $90^{\circ}$. This analysis allows to conclude that the separation distance $r$ should not exceed $0.3 \times \lambda$ for the sensor $\mathrm{B}$ :

$$
r / \lambda<0.3
$$




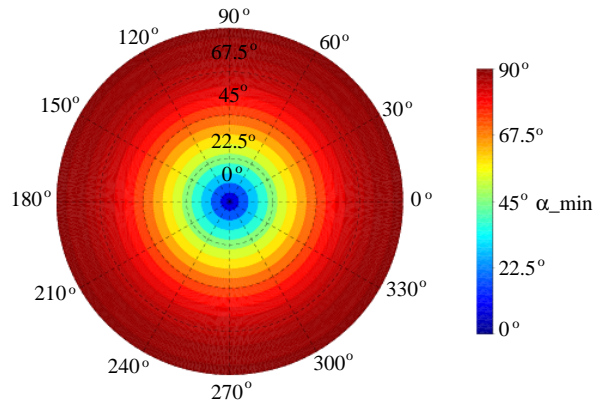

(a)

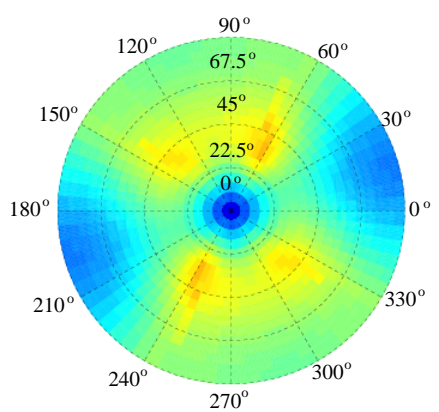

(c)

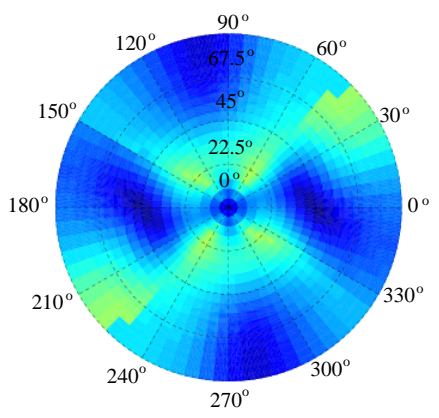

(e)

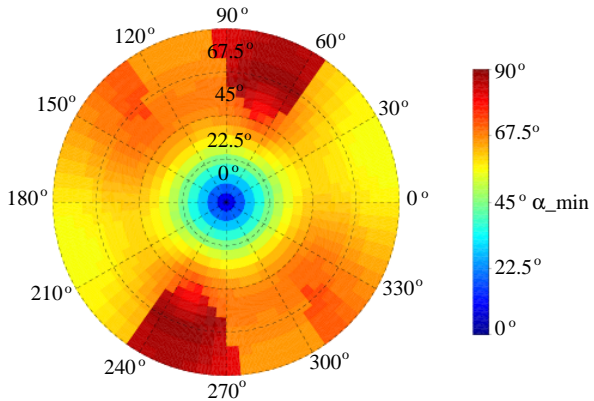

(b)

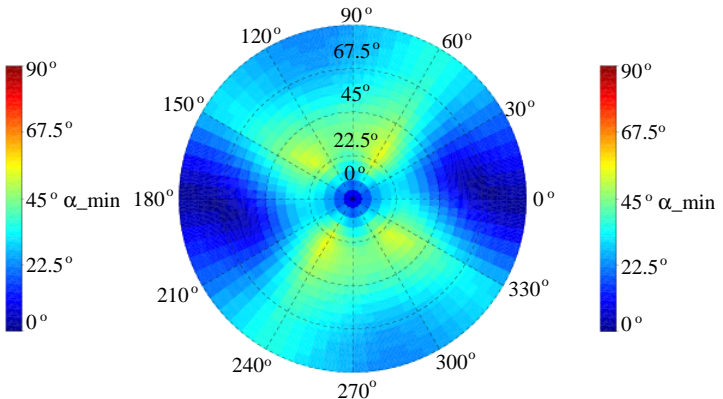

(d)

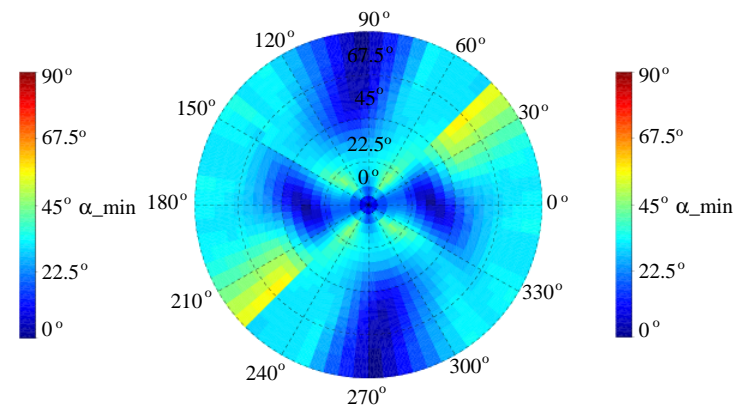

(f)

Figure 9. Theoretical 3D ambiguity spectra $\left(\alpha_{\min }\left(\phi_{1}, \theta_{1}\right)\right)$ associated with the sensor B with (a) $r=0$, (b) $r=0.1 \times \lambda$, (c) $r=0.2 \times \lambda$, (d) $r=0.3 \times \lambda$, (e) $r=0.4 \times \lambda$, (f) $r=0.5 \times \lambda$.

Table 6. Dimensions of the sensor B (in mm).

\begin{tabular}{|c|c|c|c|c|c|c|c|c|c|}
\hline$a$ & 6.74 & $c_{1}$ & 4 & $d_{1}$ & 2.2 & $e$ & 23.5 & $g$ & 100 \\
\hline$b$ & 19 & $c_{2}$ & 3.5 & $d_{2}$ & 2.2 & $f$ & 25 & & \\
\hline
\end{tabular}

Thus the distance $r$ is set to $\lambda / 4$ to avoid ambiguous angles as shown in Figure 11(a).

The sensor B is modeled using HFSS. The dimensions of the antenna (two half-loops and one linear monopole) are given in Figure 10 and in Table 6 . The operating frequency has been set to $2.4 \mathrm{GHz}$. A metallic disc is placed at the bottom of the sensor B. Its diameter is fixed to $350 \mathrm{~mm}(\approx 2.8 \lambda)$.

A new ambiguity analysis is carried out with the steering vector obtained from full wave electromagnetic simulation. This analysis confirms that for $r=\lambda / 4$ there is no ambiguous angle with this realistic vector sensor (see Figure 11(b)). The metallic disc at the bottom of the sensor B alters the ambiguity spectrum by reducing the received power for elevation angles close to $22.5^{\circ}$. However, the resulting 3D ambiguity spectrum is close to the theoretical results for other directions of arrival. 

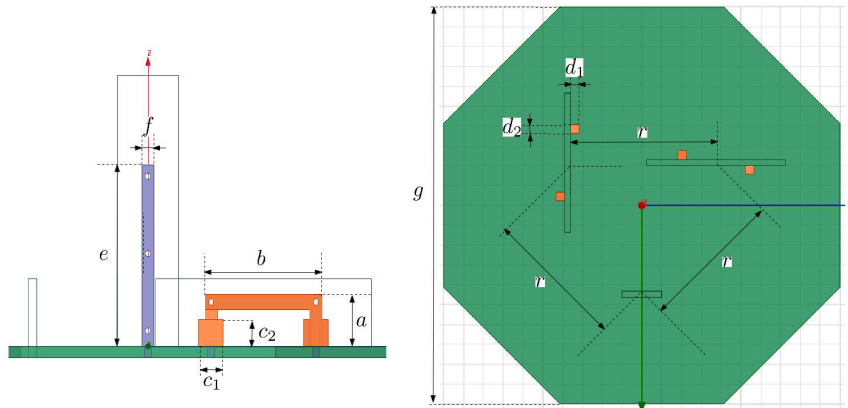

Figure 10. Vector sensor B combining three distributed radiating elements: two half-loops and one linear monopole.

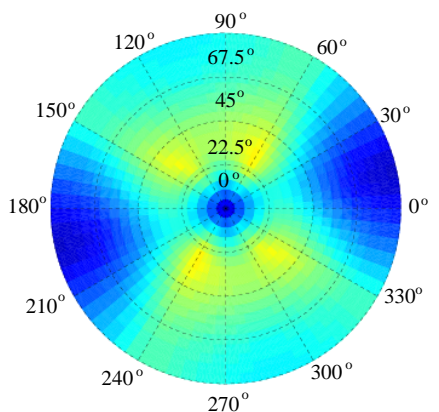

(a)

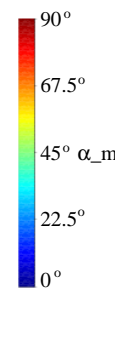

$\alpha$

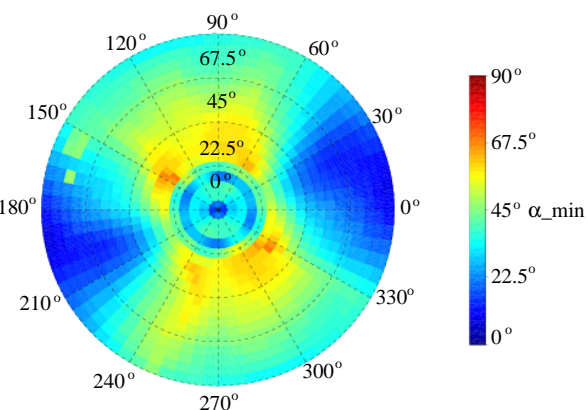

(b)

Figure 11. The 3D ambiguity spectrum $\left(\alpha_{\min }\left(\phi_{1}, \theta_{1}\right)\right)$ in the half-space with $r=\lambda / 4$ associated with (a) the theoretical sensor B, (b) the simulated sensor B.

Table 7. Mean DoA estimation error - Simulation results by using the sensor A and the sensor B $\mathrm{RSNR}=15 \mathrm{~dB}, N=100$ and $I T=100$.

\begin{tabular}{|c|c|c|c|c|c|}
\hline$\phi$ & $\theta$ & $\begin{array}{c}\text { Sensor A } \\
\text { Mean } \phi \text { error }\end{array}$ & $\begin{array}{c}\text { Sensor A } \\
\text { Mean } \theta \text { error }\end{array}$ & $\begin{array}{c}\text { Sensor B } \\
\text { Mean } \phi \text { error }\end{array}$ & $\begin{array}{c}\text { Sensor B } \\
\text { Mean } \theta \text { error }\end{array}$ \\
\hline $0^{\circ}$ & $45^{\circ}$ & $0.1^{\circ}$ & $0.1^{\circ}$ & $0.45^{\circ}$ & $0.3^{\circ}$ \\
\hline $45^{\circ}$ & $10^{\circ}$ & $0.2^{\circ}$ & $5.4^{\circ}$ & $0.7^{\circ}$ & $0.65^{\circ}$ \\
\hline $45^{\circ}$ & $80^{\circ}$ & $2.1^{\circ}$ & $4.8^{\circ}$ & $1.25^{\circ}$ & $4.2^{\circ}$ \\
\hline $55^{\circ}$ & $20^{\circ}$ & $0.8^{\circ}$ & $1.7^{\circ}$ & $0.45^{\circ}$ & $0^{\circ}$ \\
\hline $90^{\circ}$ & $80^{\circ}$ & $2.2^{\circ}$ & $5.7^{\circ}$ & $0.8^{\circ}$ & $5.2^{\circ}$ \\
\hline $95^{\circ}$ & $40^{\circ}$ & $0.1^{\circ}$ & $0.5^{\circ}$ & $0.5^{\circ}$ & $0.4^{\circ}$ \\
\hline $130^{\circ}$ & $60^{\circ}$ & $0.2^{\circ}$ & $0.4^{\circ}$ & $0.15^{\circ}$ & $1.35^{\circ}$ \\
\hline $135^{\circ}$ & $90^{\circ}$ & $4.5^{\circ}$ & $3^{\circ}$ & $1.75^{\circ}$ & $2.3^{\circ}$ \\
\hline $180^{\circ}$ & $45^{\circ}$ & $0.15^{\circ}$ & $0.35^{\circ}$ & $0.25^{\circ}$ & $0.3^{\circ}$ \\
\hline $225^{\circ}$ & $10^{\circ}$ & $0.05^{\circ}$ & $5.3^{\circ}$ & $0.15^{\circ}$ & $0.1^{\circ}$ \\
\hline $270^{\circ}$ & $45^{\circ}$ & $0.2^{\circ}$ & $0.5^{\circ}$ & $0.1^{\circ}$ & $0.25^{\circ}$ \\
\hline $315^{\circ}$ & $80^{\circ}$ & $2.35^{\circ}$ & $5.3^{\circ}$ & $0.6^{\circ}$ & $3.75^{\circ}$ \\
\hline
\end{tabular}

\subsection{Comparison of the DoA Estimation Accuracy between the Sensor A and the Sensor B}

The DoA estimation accuracy of the sensor B is computed for a RSNR of $15 \mathrm{~dB}$ and $10 \mathrm{~dB}$ and compared with one obtained by using the sensor A. The calibration matrix computed with the same process such as in the Section 3.3 is imported into the MUSIC algorithm. The number of snapshots $(N)$ and iterations 
Table 8. Mean DoA estimation error - Simulation results by using the sensor A and the sensor B $\mathrm{RSNR}=10 \mathrm{~dB}, N=100$ and $I T=100$.

\begin{tabular}{|c|c|c|c||c|c|}
\hline$\phi$ & $\theta$ & $\begin{array}{c}\text { Sensor A } \\
\text { Mean } \phi \text { error }\end{array}$ & $\begin{array}{c}\text { Sensor A } \\
\text { Mean } \theta \text { error }\end{array}$ & $\begin{array}{c}\text { Sensor B } \\
\text { Mean } \phi \text { error }\end{array}$ & $\begin{array}{c}\text { Sensor B } \\
\text { Mean } \theta \text { error }\end{array}$ \\
\hline $0^{\circ}$ & $45^{\circ}$ & $1.7^{\circ}$ & $2.75^{\circ}$ & $2.8^{\circ}$ & $2.8^{\circ}$ \\
\hline $45^{\circ}$ & $10^{\circ}$ & $4.65^{\circ}$ & $7.2^{\circ}$ & $3.95^{\circ}$ & $1.6^{\circ}$ \\
\hline $45^{\circ}$ & $80^{\circ}$ & $6.55^{\circ}$ & $6.8^{\circ}$ & $3.5^{\circ}$ & $6.3^{\circ}$ \\
\hline $55^{\circ}$ & $20^{\circ}$ & $13.45^{\circ}$ & $3.25^{\circ}$ & $4.35^{\circ}$ & $0.15^{\circ}$ \\
\hline $90^{\circ}$ & $80^{\circ}$ & $5.35^{\circ}$ & $7.15^{\circ}$ & $2.6^{\circ}$ & $6.8^{\circ}$ \\
\hline $95^{\circ}$ & $40^{\circ}$ & $1.4^{\circ}$ & $2.8^{\circ}$ & $1.45^{\circ}$ & $1.65^{\circ}$ \\
\hline $130^{\circ}$ & $60^{\circ}$ & $2^{\circ}$ & $2.65^{\circ}$ & $2^{\circ}$ & $6.6^{\circ}$ \\
\hline $135^{\circ}$ & $90^{\circ}$ & $9.3^{\circ}$ & $6.3^{\circ}$ & $4.85^{\circ}$ & $3.95^{\circ}$ \\
\hline $180^{\circ}$ & $45^{\circ}$ & $1.05^{\circ}$ & $2.3^{\circ}$ & $1.25^{\circ}$ & $2.15^{\circ}$ \\
\hline $225^{\circ}$ & $10^{\circ}$ & $3.2^{\circ}$ & $7.35^{\circ}$ & $1.7^{\circ}$ & $0.7^{\circ}$ \\
\hline $270^{\circ}$ & $45^{\circ}$ & $1^{\circ}$ & $2.55^{\circ}$ & $0.8^{\circ}$ & $2.25^{\circ}$ \\
\hline $315^{\circ}$ & $80^{\circ}$ & $6.15^{\circ}$ & $6.65^{\circ}$ & $3.5^{\circ}$ & $6.5^{\circ}$ \\
\hline
\end{tabular}

Table 9. Simulated coupling between the antenna elements of the sensor A.

\begin{tabular}{|c|c|c|c|}
\hline Sensor $\mathrm{A}$ & $E_{z}$ monopole & $H_{x}$ half-loop & $H_{y}$ half-loop \\
\hline$E_{z}$ monopole & $X$ & $-7.7 \mathrm{~dB}$ & $-7.7 \mathrm{~dB}$ \\
\hline$H_{x}$ half-loop & $-7.7 \mathrm{~dB}$ & $X$ & $-14.7 \mathrm{~dB}$ \\
\hline$H_{y}$ half-loop & $-7.7 \mathrm{~dB}$ & $-14.7 \mathrm{~dB}$ & $X$ \\
\hline
\end{tabular}

Table 10. Simulated coupling between the antenna elements of the sensor B.

\begin{tabular}{|c|c|c|c|}
\hline Sensor B & $E_{z}$ monopole & $H_{x}$ half-loop & $H_{y}$ half-loop \\
\hline$E_{z}$ monopole & $X$ & $-20.8 \mathrm{~dB}$ & $-15.5 \mathrm{~dB}$ \\
\hline$H_{x}$ half-loop & $-20.8 \mathrm{~dB}$ & $X$ & $-35.6 \mathrm{~dB}$ \\
\hline$H_{y}$ half-loop & $-15.5 \mathrm{~dB}$ & $-35.6 \mathrm{~dB}$ & $X$ \\
\hline
\end{tabular}

(IT) are both set to 100 . The results are given in Table 7 and in Table 8.

The simulation results show that the sensor B is more accurate than the sensor A. For high RSNR $(\geq 15 \mathrm{~dB})$, the maximum estimation error in the half-space for the sensor B is $1.65^{\circ}$ in azimuth and $5.2^{\circ}$ in elevation and $4.5^{\circ}$ and $5.7^{\circ}$ for the sensor A. When the RSNR is $10 \mathrm{~dB}$, the highest error is $4.85^{\circ}$ in azimuth and $6.8^{\circ}$ in elevation for the sensor B and $13.45^{\circ}$ and $7.35^{\circ}$ for the sensor A. Such improvement of the accuracy is due to the additional information on DoA provided by the spatial phase shift but also to the reduction of the coupling effect, as shown in Table 9 and in Table 10 for the sensor A and the sensor B respectively. The mutual coupling reduces the dynamic of the power received by each antenna port and consequently alters the DoA estimation sensitivity. Thus the sensor A is found to be less robust to the noise level than the sensor B.

\section{CONCLUSION}

In this work, two new designs of vector sensor have been proposed to estimate the directions of arrival in the 3D space (azimuth and elevation angles) of TM electromagnetic signals. The two sensors are composed of three antenna elements only: two orthogonal half-loops and one linear monopole. Each design uses a metallic plate as antenna support in view of embedded applications. One sensor, called sensor A, uses co-located elements while the other one, called sensor B, is based on distributed antenna elements. The accuracy of the DoA estimation with the sensor A has been computed through full 
wave electromagnetic simulations and by using the MUSIC algorithm. A prototype of this antenna has been manufactured for validation purpose with measurements performed in an anechoic chamber. The sensor B has been studied with the same simulation process. A 3D ambiguity analysis has been carried out to determine the separation distance between the antenna elements without causing ambiguous estimations. This distance is found to be close to $\lambda / 3$. The DoA estimation with the sensor B has been found more accurate than one obtained by using the sensor A. It is due to the reduction of the EM coupling and the additional information on the DoA provided by the spatial phase shift resulting from the spatial distribution of the antenna elements of the sensor B.

\section{ACKNOWLEDGMENT}

The authors wish to acknowledge M. Christophe IMBERT, from Rockwell Collins, for providing his support in this work.

\section{REFERENCES}

1. Tuncer, T. E. and B. Friedlander, Classical and Modern Direction-of-arrival Estimation, Elsevier, Ed., Academic Press, 2009.

2. Gething, P. J. D., Radio Direction Finding and Superresolution, P. Peregrinus Ltd. on Behalf of the Institution of Electrical Engineers, 1991.

3. Hatke, G. F., "Conditions for unambiguous source location using polarization diverse arrays," Asilomar Conference on Signals, Systems and Computers, 1365-1369, Nov. 1993.

4. Lo Monte, L., B. Elnour, and D. Erricolo, "Distributed 6D vector antennas design for direction of arrival applications," International Conference on Electromagnetics in Advanced Applications, ICEAA, 431-434, Sep. 2007.

5. Lo Monte, L., B. Elnour, D. Erricolo, and A. Nehorai, "Design and realization of a distributed vector sensor for polarization diversity applications," International Waveform Diversity and Design Conference, 358-361, Jun. 2007.

6. Sun, L., C. Li, Y. Lu, and G. Ou, "Distributed vector sensor cross product added with music for direction of arrival estimation," Asia-Pacific Symposium on Electromagnetic Compatibility, APEMC, 1354-1357, Apr. 2010.

7. Chabory, A., C. Morlaas, and B. Souny, "Efficiency characterization of vector-sensor antennas with distributed elements for 3D direction finding," IEEE-APS Topical Conference on Antennas and Propagation in Wireless Communications, 819-822, Sep. 2011.

8. See, C. M. S. and A. Nehorai, "Source localization with distributed electromagnetic component sensor array processing," 7th International Symposium on Signal Processing and Its Applications, Vol. 1, 177-180, Jul. 2003.

9. Wong, K. T. and X. Yuan, "vector cross-product direction-finding' with an electromagnetic vectorsensor of six orthogonally oriented but spatially noncollocating dipoles/loops," IEEE Transactions on Signal Processing, Vol. 59, No. 1, 160-171, Jan. 2011.

10. Malloy, N., "Analysis and synthesis of general planar interferometer arrays," IEEE International Conference on ICASSP Acoustics, Speech, and Signal Processing, Vol. 8, 352-355, Apr. 1983.

11. Nehorai, A. and E. Paldi, "Vector-sensor array processing for electromagnetic source localization," IEEE Transactions on Signal Processing, Vol. 42, No. 2, 376-398, Feb. 1994.

12. Yuan, X., K. T. Wong, Z. Xu, and K. Agrawal, "Various compositions to form a triad of collocated dipoles/loops, for direction finding and polarization esitmation," IEEE Sensors Journal, Vol. 12, No. 6, 1763-1771, Jun. 2012.

13. Hirari, M. and M. Hayakawa, "DoA estimation using blind separation of sources," Proceedings of the IEEE Signal Processing Workshop on Higher-Order Statistics, 311-315, Jul. 1997.

14. Mir, H. S., J. D. Sahr, and C. M. Keller, "Source localization using airborne vector sensors," IEEE International Conference on Acoustics, Speech, and Signal Processing, ICASSP, Vol. 4, iv/1033iv/1036, Mar. 2005. 
15. Mir, H. S. and J. D. Sahr, "Passive direction finding using airborne vector sensors in the presence of manifold perturbations," IEEE Transactions on Signal Processing, Vol. 55, No. 1, 156-164, Jan. 2007.

16. Wong, K. T. and A. K. Y. Lai, "Inexpensive upgrade of base-station dumb antennas by two magnetic loops for 'blind' adaptive downlink beamforming," IEEE Antennas and Propagation Magazine, Vol. 47, No. 1, 189-193, Feb. 2005.

17. Lominé, J., C. Morlaas, and H. Aubert, "Method for vector sensor design based on a spherical mode approach for 3D DoA estimation," 7th European Conference on Antennas and Propagation, EuCAP, 237-241, Apr. 2013.

18. Appadwedula, S. and C. M. Keller, "Direction-finding results for a vector sensor antenna on a small UAV," Fourth IEEE Workshop on Sensor Array and Multichannel Processing, 74-78, Jul. 2006.

19. Slater, M. J., C. D. Schmitz, M. D. Anderson, D. L. Jones, and J. T. Bernhard, "Demonstration of an electrically small antenna array for UHF direction-of-arrival esitmation," IEEE Transactions on Antennas and Propagation, Vol. 61, No. 3, 1371-1377, Mar. 2013.

20. Schmidt, R. O., "Multiple emitter location and signal parameter estimation," IEEE Transactions on Antennas and Propagation, Vol. 34, 276-280, Mar. 1986.

21. Roy, R. and T. Kailath, "ESPRIT-estimation of signal parameters via rotational invariance techniques," IEEE Transactions on Acoustics, Speech and Signal Processing, Vol. 37, No. 7, 984995, Jul. 1989.

22. Chandran, S., Advances in Direction-of-arrival Estimation, Artech House, Incorporated, 2005.

23. Lemma, A. N., E. F. Deprettere, and A. J. van der Veen, "Experimental analysis of antenna coupling for high-resolution DoA estimation algorithms," 2nd IEEE Workshop on Signal Processing Advances in Wireless Communications, SPAWC, 362-365, May 1999.

24. Wang, G., H. Tao, J. Su, X. Guo, C. Zeng, and L. Wang, "Mutual coupling calibration for electromagnetic vector sensor array," 10th International Symposium on Antennas, Propagation EM Theory, ISAPE, 261-264, Oct. 2012.

25. Khallaayoun, A., R. J. Weber, and Y. Huang, "A blind iterative calibration method for high resolution DoA estimation," Military Communications Conference, MILCOM, 199-204, Nov. 2011.

26. Tan, C. M., S. E. Foo, M. A. Beach, and A. R. Nix, "Ambiguity in MUSIC and ESPRIT for direction of arrival estimation," Electronics Letters, Vol. 38, No. 24, 1598-1600, Nov. 2002.

27. Eric, M., A. Zejak, and M. Obradovic, "Ambiguity characterization of arbitrary antenna array: Type I ambiguity," IEEE 5th International Symposium on Spread Spectrum Techniques and Applications, Vol. 2, 399-403, Sep. 1998.

28. Spielman, D., A. Paulraj, and T. Kailath, "Performance analysis of the MUSIC algorithm," IEEE International Conference on Acoustics, Speech, and Signal Processing, ICASSP, Vol. 11, 1909-1912, Apr. 1986. 